\title{
Science Policy
}

National Cancer Institute

\section{Source}

National Cancer Institute. Science Policy. NCI Thesaurus. Code C19025.

The prioritizing of science projects by a government 